Research Paper

\title{
Effects of a Pre-workout Supplement on Lean Mass, Muscular Performance, Subjective Workout Experience and Biomarkers of Safety
}

\author{
A. William Kedia ${ }^{1}$, Jennifer E. Hofheins ${ }^{1}$, Scott M. Habowski ${ }^{1}$, Arny A. Ferrando ${ }^{2}$, M. David Gothard ${ }^{3}$, and \\ Hector L. Lopez ${ }^{\natural}$ \\ 1. The Center for Applied Health Sciences, 4302 Allen Road, Suite 120, Stow, OH 44224, USA \\ 2. Center for Translational Research in Aging and Longevity, University of Arkansas for Medical Sciences, 4305 W. Markham St Slot 806 , \\ Little Rock, AR 72205, USA \\ 3. BIOSTATS, 501 Wood Street North, East Canton, Ohio 44730, USA
}

$\triangle$ Corresponding author: hl@appliedhealthsciences.org

(C) Ivyspring International Publisher. This is an open-access article distributed under the terms of the Creative Commons License (http:/ / creativecommons.org/ licenses/by-nc-nd/3.0/). Reproduction is permitted for personal, noncommercial use, provided that the article is in whole, unmodified, and properly cited.

Received: 2013.07.03; Accepted: 2013.12.17; Published: 2014.01.02

\begin{abstract}
In this prospective, randomized, double-blind, trial conducted in two parts, we examined the effects of a multi-ingredient pre-exercise workout supplement blend of creatine, betaine and a dendrobium extract (MMP) on safety, performance, and body composition in healthy men and women undergoing a supervised program of resistance exercise. Part I was an acute hemodynamic safety study wherein forty young, healthy men and women $(26.2 \pm 5.3$ years, $70.4 \pm 3.3$ inches, 83.7 $\pm 14.9 \mathrm{~kg}, 26.0 \pm 3.2 \mathrm{~kg} \bullet \mathrm{m}^{-2}$ ) ingest one dose of either the MMP or comparator in a randomized, double-blind, comparator controlled, crossover fashion before having their resting heart rate, blood, ECG and comprehensive blood chemistry and blood counts completed. Systolic (SBP) and diastolic (DBP) blood pressures were generally raised $(3.0-5.4 \mathrm{~mm} \mathrm{Hg}, \mathrm{p}<0.01)$ following supplementation with MPP whereas in the comparator group SBP was marginally reduced by 0.3 to $I .2$ $\mathrm{mm} \mathrm{Hg}, \mathrm{p}>0.05$ at all time points) and DBP was increased $(3.0-3.9 \mathrm{~mm} \mathrm{Hg}, \mathrm{p}<0.05$ at all time points). No changes in EKG-corrected QT interval were observed, and no serious adverse events were reported. Part 2 was a six-week training study wherein forty-three young, healthy men and women $\left(24.3 \pm 2.9\right.$ years, $70.5 \pm 3.1$ inches, $\left.83.8 \pm 9.6 \mathrm{~kg}, 26.1 \pm 2.7 \mathrm{~kg} \bullet \mathrm{m}^{-2}\right)$ supplemented with daily pre-workout doses of either the MPP or a comparator in a randomized, double-blind, comparator-controlled fashion while following a standardized resistance training program for six weeks. MPP and the comparator were isocaloric and delivered the same amount of caffeine. Significant improvements in visual analog scale (VAS) scores for energy $(p<0.024)$ and concentration $(p<0.04 \mathrm{I})$ were found along with consistently higher levels of focus accompanied by less fatigue when MPP was consumed in comparison to comparator during upper body muscular strength-endurance tests at weeks 3 and 6. MPP supplementation for 6 weeks did not improve dual-energy $x$-ray absorptiometry (DEXA) measures of body composition or objective assessments of exercise performance. Overall, MPP use and administration was well tolerated. Self-reported scores for energy and concentration were significantly greater. Over a six-week training and supplementation period, MPP use was not associated with improvements in performance or body composition. Future studies should confirm these effects over a more prolonged training period.
\end{abstract}

Key words: pre-workout, dietary supplement, dendrobium, caffeine, safety, creatine, botanicals, sports nutrition 


\section{Introduction}

Supplementation with various over-the-counter dietary supplements and "nutrient timing" are two popular approaches used by athletes to aid in performance and recovery $[1,2]$. The pre-workout time period has become an extremely popular window of nutrient administration where athletes ingest various combinations of key ingredients in an attempt to maximize training adaptations and promote recovery. While a near infinite combination of ingredients can exist, most multi-nutrient formulations contain a combination of key ingredients such as creatine monohydrate, amino acids, betaine, selected botanicals and plant extracts, carbohydrates, and caffeine [3-6]. For example, Kraemer et al. required research participants to ingest isocaloric doses of either a comparator or a multi-nutrient supplement for seven days prior to completing two consecutive days of heavy resistance training. Multi-nutrient supplementation improved vertical jump power, repetitions performed at $80 \%$ of their one-repetition maximum (1RM) and also favorably impacted hormonal changes [4]. Additionally, Spillane et al. had 19 non-trained males follow a resistance training program for four weeks while consuming isocaloric combinations of two different multi-nutrient products (NO-Shotgun and NO-Synthesize, Vital Pharmaceuticals, Davie, FL). Greater improvements in fat mass and fat-free mass were found to occur in addition to greater increases in myofibrillar protein content as well as the MHC isoforms [6]. Similarly, Fukuda and Smith both reported on data from a large group of college-aged men and women who consumed a combination of amino acids, adaptogens, caffeine, and creatine after one dose and one bout of high-intensity interval treadmill running as well as after three weeks of taking the supplement and performing intervals. Greater amounts of work were able to be performed when the multi-nutrient combination was ingested after just one dose [3] and greater improvements in body composition were noted after supplementation and training [5].

As highlighted above, many different nutritional ingredients could be included in multi-nutrient formulations, but some of the most popular ingredients include creatine, betaine, and caffeine. Over the past twenty years, no dietary supplement has received as much scientific scrutiny as creatine. Today, creatine remains to be one of the most popular natural supplements due to its ability to increase endogenous phosphocreatine stores and thus serve as an aid in ATP resynthesis $[7,8]$. A number of original investigations have also documented its ability to help increase strength and performance while also improving many components of body composition alone [9] or in combination with carbohydrates and protein [10]. Many review articles have been published that detail the safety and efficacy of creatine supplementation in humans (for examples, see [7] and [11]).

Betaine (trimethyglycine: TMG) is another widely used dietary supplement that has also been shown to enhance exercise performance in resistance-trained individuals. A recent randomized, double-blind study reported significant increases in total repetitions $(\mathrm{P}=0.01)$ and total volume load $(\mathrm{P}=0.02)$ in a 10 -set bench press protocol with betaine supplementation [12]. Mechanistically, significant reductions $(\mathrm{P}=0.01)$ in muscle tissue oxygen saturation during the bench press protocol in the betaine supplementation group were found, suggesting that enhanced muscle oxygen consumption and/or extraction might be the result of betaine use.

Caffeine is a common component of exercise-related dietary supplements largely because of its known positive central nervous system (CNS) effects including: an attenuation of the perception of effort during fatiguing exercise, stimulation of fatty acid mobilization and various direct effects on muscle function [13]. In addition, caffeine administration is well-known to exert both favorable physical and mental outcomes including increases in endurance performance [14] as well as increases in alertness, mental focus and cognition [15]. Moreover, caffeine stimulates the CNS by antagonizing adenosine receptors, thereby inhibiting the negative effects adenosine induces on neurotransmission, arousal, and pain perception $[16,17]$. In short, caffeine is commonly used and accepted for its ability to promote wakefulness, enhance focus and concentration and to prevent the central perception of fatigue [12].

While ingredients such as creatine, betaine and caffeine are well established, the MPP tested in the present study also contained the proprietary product Dendrobex $^{\mathrm{TM}}$ (dendrobium extract; stems of genus Dendrobium [Orchidaceae]), an herbal medicine (Dendrobii Herba) traditionally used in Eastern Asia that might have blood pressure-lowering effects while serving as a stimulant and a neurotropic (See figure 1 for supplement facts panel) [18].

The present research was a two-part study whereby the first study was an acute (single dose), cross-over experiment to assess the effects of MPP on markers of safety of the pre-workout supplement Craze $^{\mathrm{TM}}$ (Driven Sports, NY; multi-ingredient performance product; MPP). The second study was a six-week, randomized, double-blind, parallel-group experiment in healthy men and women that determined the impact effects of MPP on: 1) physical performance, 2) body composition, and 3) mood, focus, cognition and alertness. As far as the authors are 
aware, this is the first in vivo trial in humans of a pre-workout product containing Dendrobex ${ }^{\mathrm{TM}}$ or any product containing components of dendrobium.

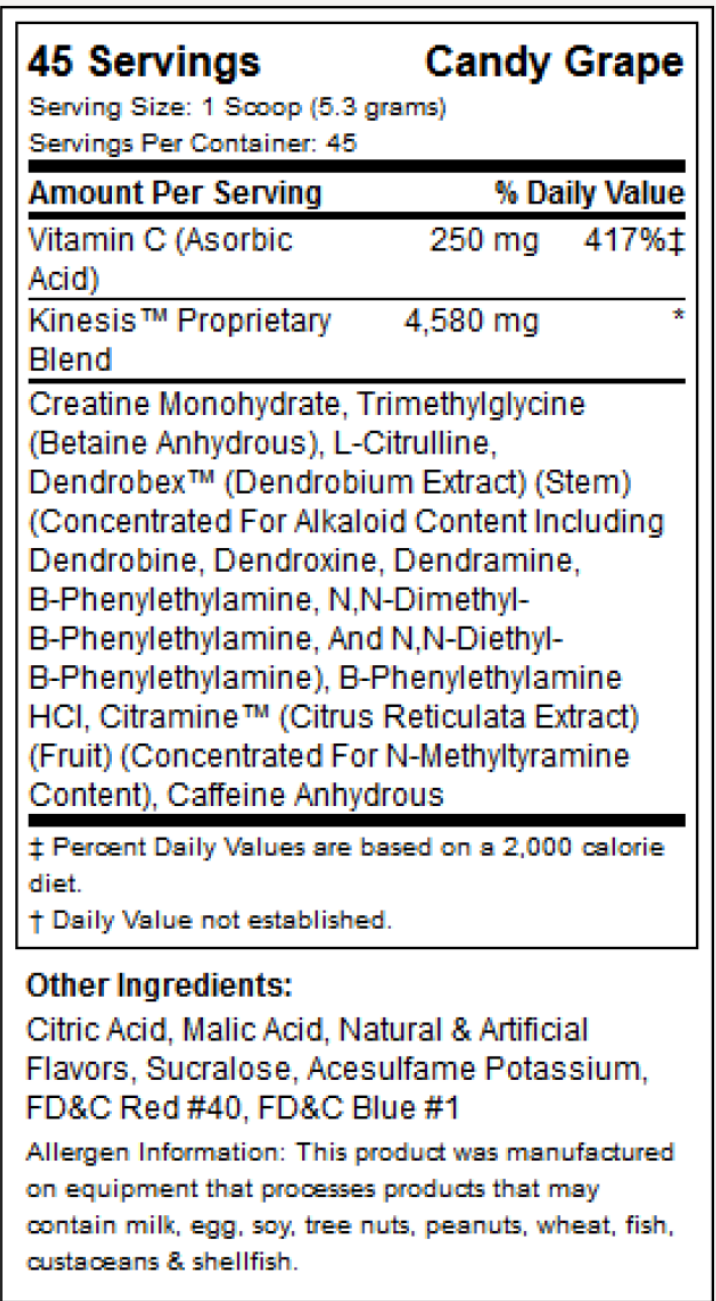

Figure I. MPP supplement facts panel.

\section{METHODS}

\section{Study Design: Part I (Acute Hemodynamic Safety Study)}

\section{Subjects}

Forty healthy men and women (26.2 \pm 5.3 years, $70.4 \pm 3.3$ inches, $83.7 \pm 14.9 \mathrm{~kg}, 26.0 \pm 3.2 \mathrm{~kg}^{-2}$ ) provided written informed consent to participate in this institutional review board (IRB)-approved clinical trial prior to commencing any study-related activities and agreed to follow all prescribed exercise and supplementation regimens. The study protocol was approved by a private IRB (Integreview, Austin, TX, Protocol \# DS-CRZ-001, approval date: 6/8/2012 and Protocol \# CHR-CRZ-001, approval date: 9/4/2012). During their initial visit, participants completed medical history paperwork and were screened for eligibility by the same study physician. Participants were classified as healthy if they were normotensive (resting systolic/diastolic blood pressure [SBP/DBP], $<140 /<90 \mathrm{mmHg}$ ), had a normal resting heart rate $(<90 \mathrm{bpm})$ and basal body temperature $\left(<99^{\circ} \mathrm{F}\right.$ $\left.\left[<37.2^{\circ} \mathrm{C}\right]\right)$. Participants were excluded if they had any metabolic disorder including known electrolyte abnormalities, diabetes, thyroid disease, hypogonadism, or other endocrine disorder and history of hepatic, renal, musculoskeletal, autoimmune, or neurologic disease. Exclusion criteria also included subjects with history of heart disease, hypertension, psychiatric disorders, cancer, benign prostatic hypertrophy, caffeine sensitivity, gastric ulcer, gastroesophegal reflux disease, or any other medical disorder deemed unsuitable for inclusion in the study by the investigators. Participants who reported a history of taking creatine, betaine or other dietary supplements were only allowed entry if they had not taken or had refrained from taking any dietary supplements containing these ingredients for at least 30 days (excluding a multi-vitamin/mineral). Participants who regularly consumed caffeine were neither excluded nor restricted and were instructed to continue with their normal daily intake. Participants currently prescribed any thyroid, antihyperlipidemic, glucose controlling, antihypertensive, anticoagulant, or androgenic medications, nitrates/nitrate derivatives, or phosphodiesterase (PDE)-5 inhibitors were disallowed. Subjects who had taken anabolic steroids, growth hormone, insulin-like growth factor (IGF)-1, or other hormone medication including oral contraceptives during the previous 12 months were also disallowed, as were smokers and those with orthopedic limitations or injuries. Female subjects who were pregnant, trying to become pregnant, or nursing were also excluded.

Diet

Subjects presented a baseline 24-hour diet recall to a licensed, registered dietician. Copies of this recall were provided to each participant and they were instructed to replicate their food and fluid intake as much as possible for each subsequent study visit. No strenuous physical activity was allowed for 48 hours before each visit. All dietary information was analysed for average energy and macronutrient content as well as macronutrient ratio using NutriBase IV software (CyberSoft Inc., Phoenix, AR).

\section{Supplementation}

In a randomized, double-blind, comparator-controlled, crossover fashion, participants were instructed to consume one dose of their assigned 
supplement after observing a modified fast of 8-10 hours. Specifically, participants were instructed to fast overnight and to consume no food or fluid with caloric content after midnight prior to each test. All tests were scheduled between hours of 0600 and 1000 to limit within-subject variation. Participants were instructed to ingest each dose with 8-16 fluid ounces of cool water per manufacturer instructions. The multi-ingredient performance product (MPP) was trademarked as Craze ${ }^{\mathrm{TM}}$ (Driven Sports, NY) and a copy of the supplement facts label is shown in figure 1. The comparator was an isocaloric beverage of similar taste, color, smell and texture with identical caffeine intake. Craze ${ }^{\mathrm{TM}}$ and comparator were produced in accordance with current Good Manufacturing Practices (cGMP) in a United States Food and Drug Administration (FDA) registered facility. Prior to production, all raw materials were tested for purity and potency. A sample of the lot from the comparator and MPP finished product was tested by an independent third party laboratory to verify ingredient label claims and was shown to be within +/- $2 \%$ for the botanical actives and had a $10 \%$ overage on ascorbic acid (vitamin C) of the actual formulation for the main bioactive ingredients (Samples: Lot \# 1208079 " $\mathrm{A}$ " and "B" for the acute study-part 1 and Lot \# 1202485 " $A$ " and "B" for the 6 week study-part 2). Samples from both MPP and comparator lots utilized in this trial were also analyzed via gas-chromatography-mass-spectrometry (GC/MS), using analytical reference standards for a variety of potential amphetamine and controlled substance adulterants by a $3^{\text {rd }}$ party Drug Enforcement Administration (DEA) registered, cGMP and ISO 17025 compliant analytical laboratory (Lab ID\# 12-0429-06 and 12-00341-03) with no detection, except for caffeine at $16.4 \mathrm{mg} / \mathrm{g}$ and $21.9 \mathrm{mg} / \mathrm{g}$, respectively. An additional sample of the aforementioned MPP lot was ex post facto analyzed for presence of $\mathrm{N}$, a-diethylphenylethylamine via liquid-chromatography-mass-spectrometry (LC-MS) of reference standard PEA and its analogues by a $3^{\text {rd }}$ party, U.S. Food and Drug Administration FDA-registered, cGMP compliant and DEA-licensed laboratory (Lab ID\# 061913DR1526) with no detection.

A random order of supplementation administration was followed and both testing conditions were separated by a minimum of three days. Participants were allowed to continue normal activities, but were restricted from strenuous exercise for 24 hours prior to the second testing session.

\section{Testing Protocol}

At baseline, subjects completed a health history questionnaire and underwent physical examinations by the same study physician. Blood pressure and heart rate assessment occurred at baseline (pre-ingestion) and 30, 60, 90, 120, 150, and 180 minutes post-ingestion by an automated device (Omron HEM-780, Lake Forest, IL). In addition, a standard 12-lead ECG was performed at baseline and 180 minutes post-ingestion to determine the presence any abnormalities or arrhythmias secondary to supplement ingestion. These time points were selected to capture typical half-life responses of caffeine and other alkaloids. The QTc interval, measured in milliseconds, was analyzed with guidance from the Guide for the Analysis and Review of QT/QTc Interval Data - Minister of Public Works and Government Services Canada 2010. Fridericia's formula was then employed to correct for possible R-R interval (heart rate) influence on $\mathrm{QTc}$ values $(\mathrm{QTC}=\mathrm{QT} / 3 \sqrt{\mathrm{RR}})$. Additional adjustment was performed using population specific linear modeling where QTc values were regressed on (1-RR) values using the initial baseline study time point.

Participants were required to remain in the research laboratory between evaluations and were allowed to watch television and remain in a rested state with no exertion. All blood samples were analyzed for clinical chemistry analysis (plasma glucose, blood urea nitrogen [BUN], creatinine, aspartate transaminase [AST], alanine transaminase [ALT], creatine kinase, lactate dehydrogenase, total bilirubin, alkaline phosphatase [ALP], triacylglycerides [TG], total cholesterol [TC], LDL, HDL, uric acid, sodium, potassium, total protein, albumin, globulin, iron, complete blood cells, and platelet count) using automated clinical chemistry analyzers (Quest Diagnostics, Pittsburgh, PA branch).

\section{Statistical Analyses}

All statistical analysis were completed while group assignments were still blinded. After confirming no order effects, safety data were analyzed via independent-sample Student's $t$-test for between-group comparison of change vs. baseline as well as paired-sample Student's $t$-test for within-group comparison of change vs. zero. In all analyses, a statistically significant difference was assumed when the probability of a type I error was $<0.05$ $(\mathrm{P}<0.05)$. Statistical analyses were carried out using SPSSv21.0 software.

\section{Study Design: Part 2 (6-week Efficacy Study)}

\section{Subjects}

Healthy men and women aged $18-45$ years (24.3 \pm 2.9 years, $70.5 \pm 3.1$ inches, $83.8 \pm 9.6 \mathrm{~kg}, 26.1 \pm 2.7$ $\mathrm{kg} / \mathrm{m}^{2}$ ) with body fat $10-25 \%$ and body mass index 
(BMI) $\leq 30 \mathrm{~kg} / \mathrm{m}^{2}$ who had been undergoing resistance training regularly (defined as completing an average of three workouts/week) for $\geq 2$ years were eligible for inclusion. Exclusion criteria were the same as described for Part 1. The study was approved by a private IRB (Integreview, Austin, TX, Protocol \# DS-CRZ-001, approval date: 6/8/2012 and Protocol \# CHR-CRZ-001, approval date: 9/4/2012) and all subjects provided written informed consent to participate in this clinical trial prior to commencing any study-related activities.

\section{Diet}

Subjects recorded their dietary intake over a three-day period (two week days, one weekend day) according to instructions given by a licensed, registered dietician. Each subject's baseline diet was analyzed by NutriBase IV software (CyberSoft Inc., Phoenix, AR) to determine its energy and macronutrient contents. Follow-up three-day dietary records were collected and analyzed at weeks 3 and 6 to verify that eating habits remained consistent throughout the study.

\section{Resistance Training}

This study was conducted and all data were collected at the Center for Applied Health Sciences (CAHS), Stow, OH. All subjects followed a specific four-day/week workout designed by a certified strength and conditioning specialist. The workout was designed to train the upper body and lower body two times/week each on a 4-day split (upper body, lower body, upper body, lower body) with gradually increasing volume and intensity based off of the work of Kerksick [19]. The workout consisted of 10-12 exercises including but not limited to the following: bench press, lat pulldown, shoulder press, seated row, shoulder shrug, dip, biceps curl, triceps pushdown, leg press, squat, deadlift, lunge, leg curl, leg extension, and calf raise. A simple linear periodization was followed whereby participants trained using three sets of 12-15 RM loads initially and completed the program using 4-6 sets of 8 RM loads. For the bench press exercise, \%1RM load assignment was used, but for all other loads, loading was used according to repetitions maximums, pre-determined repetition ranges and following previously instructed loading rules (i.e., $2 \times 2$ rule). Using this rule, participants were instructed to increase their weight when they could perform more than two repetitions (above the target range) on two consecutive sets. Thus, progression was followed and as strength and endurance improved, training loads were increased to maintain recommended ranges. Rest periods between exercises were 1-3 minutes and between sets were 60-120 seconds.
Daily workouts were not supervised by study investigators, but study participants were given a training $\log$ to complete for each workout and each workout was signed off by a training partner or a member of the fitness staff.

\section{Supplementation}

Subjects were randomized into two groups matched for age, weight, and resistance-training experience using a double-blind approach to ingest either a multi-ingredient performance product (MPP) or a comparator. As highlighted previously, the multi-ingredient performance product (MPP) was trademarked as Craze ${ }^{\mathrm{TM}}$ (Driven Sports, NY) and a copy of the supplement facts label is figure 1 . The comparator was an isocaloric beverage of similar taste, color, smell, texture, caffeine content and packaging. All subjects ingested one serving of their prescribed supplement (MPP or comparator) and mixed it with eight fluid ounces $(240 \mathrm{~mL})$ of cold water 30 minutes prior to starting each training session. On non-training days, subjects took their allocated supplement in the mornings before or during breakfast; hence subjects in both groups took MPP or comparator product every day throughout the 6-week duration of this study. Compliance was monitored by having participants complete a supplementation log while also being required to return their empty canisters. In addition, weekly text messages and emails were sent to remind study participants of all study requirements.

\section{Testing Protocol}

Prior to pretesting, subjects were instructed not to do any strenuous exercise for $\geq 48$ hours and not consume any food or fluid with caloric contact past midnight the night before testing; most participants observed a ten hour fast. Subjects were familiarized with the experimental procedures and practiced the exercise tests prior to pre-supplementation/baseline testing. Physical activity levels and health history were determined by a standardized questionnaire. Subjects were contacted every week to determine whether they had experienced any idiosyncratic responses to the supplementation protocol; moreover, on weeks 3 and 6 subjects filled out a questionnaire to monitor individual changes in delayed-onset muscle soreness (DOMS), appetite, thirst, muscle cramping, stomach distress, and any other side effects. Additionally, vital signs such as blood pressure and heart rate were assessed at baseline and weeks 3 and 6 . Fasting blood lipids (TC, LDL, HDL, and TG), whole blood cell counts, and serum markers of hepatic/renal function (AST, ALT, BUN, creatinine, total bilirubin, ALP) were recorded at baseline and end of study (week 6) (Quest Diagnostics, Pittsburgh, PA). 
Lean mass, fat mass, and \%fat were determined by dual-energy x-ray absorptiometry (DEXA; General Electric Lunar DPX Pro) at baseline and weeks 3 and 6. All DEXA scans were performed by the same technician and analyzed by the manufacturer's software (enCORE version 13.31); reliability assessments using our device and protocol have been published previously [20]. Female subjects were measured during the early follicular phase of their menstrual cycle to control for diurnal changes in estrogen and its physiological effects (e.g., fluid retention, etc.). Briefly, subjects were positioned in the scanner according to standard procedures and remained motionless for approximately 15 minutes during scanning. DEXA segments for the upper and lower limbs and trunk were directed using standard anatomical landmarks. Percent fat was calculated by dividing fat mass by total scanned mass. Lean to fat mass ratio was computed using a simple ratio between the two values. Quality control calibration procedures were performed prior to all scans using a calibration block provided by the manufacturer. Prior to this study, we determined test-retest reliability for repeated measurements of lean mass, bone mineral content, and fat mass using this DEXA using intra-class correlation coefficients; all values were $>0.98$ [20].

On the same day as blood sampling and body composition assessment, upper body muscle strength was estimated by 1-RM bench press protocol that was adapted from standard NSCA protocols [21]. Only upper-body was assessed due to previous experience indicating that greater stability and reliability of the data is present when assessing upper body vs. lower body activities [22]. After a general warm-up of 3-5 minutes light activity (upper body ergometry) and static stretching exercises of the involved musculature, the subject performed a warm-up set of eight repetitions at approximately $50 \%$ of the perceived 1-RM followed by a set of three repetitions at $70 \%$ of the perceived 1-RM. Thereafter, the subject performed single lifts at progressively heavier weights until failure. At failure, a single lift of a weight approximately midway between the last successful and failed lift was attempted and this process was repeated until the 1-RM was determined. No more than five maximal attempts were completed in one testing session. Upper body muscle endurance was estimated by the total number of repetitions completed (repetitions to failure; RTF) during three successive sets (separated by 1-minute rest intervals) of isotonic bench press at a load equal to $100 \%$ of subjects' pretesting body weight. The aggregate or total number of repetitions performed were used for statistical determination of muscular endurance. Upper body power production (average power $[\mathrm{AP}]$, average velocity $[\mathrm{AV}]$, peak power [PP], peak velocity [PV]) was assessed during the bench press exercise using a Tendo unit. Previous studies have incorporated the use of a Tendo into their study design [23] and Stock and colleagues [24] recently published data to indicate it is a reliable means of assessment. The unit consists of a position transducer that measures the rate of linear displacement providing velocity and acceleration in addition to power production.

Subjective levels of fatigue, energy, focus, and concentration were assessed by visual analog scale (VAS) immediately before commencing each workout. Using VAS, subjects were asked to mark their feelings on a 15-cm straight line anchored with verbal cues such as "high" and "low" at each end. The validity and reliability of VAS to assess fatigue and energy have been previously established [25].

\section{Statistical Analyses}

All statistical analyses were completed while group assignments were still blinded. All collected data were first screened to meet normality and sphericity assumptions. Between-group differences in body composition, muscular performance, and safety markers were assessed by analysis of covariance (ANCOVA) using baseline scores as the covariate. When significant interaction effects were found, main effects were decomposed using independent samples t-test for between group determinations and paired sample t-tests for within-group assessments. In all analyses, a statistically significant difference was assumed when the probability of a type I error was $<0.05(\mathrm{P}<0.05)$. Statistical analyses were carried out using SPSSv21.0 software.

\section{RESULTS}

\section{Part I: Acute Hemodynamic Safety Study}

\section{Subjects}

A total of 40 subjects were recruited for part 1. Twenty-two participants were randomized to receive the comparator first, while 18 received the MPP during the first experimental trial. Baseline demographic information for both the MPP and PLA groups are provided in table 1 .

\section{Safety Profiles}

Following supplementation, SBP and DBP were generally raised in the MPP group whereas SBP was marginally elevated and DBP reduced in the comparator group (Table 1 ). In subjects taking MPP, statistically significant $(3.4-5.4 \mathrm{~mm} \mathrm{Hg}, \mathrm{p}<0.01)$ increases in SBP were noted at 30, 60, 90, and 120 minutes post-ingestion and of DBP at 60, 90, and 120 minutes post-ingestion $(2.3-4.2 \mathrm{~mm} \mathrm{Hg}, \mathrm{p}<0.05)$. Following 
ingestion of comparator, no significant changes of SBP were noted at 30, 60, 90, and 120 minutes post-ingestion whereas DBP was significantly increased at 30,60, 90, and 120 minutes post-ingestion (3.0-3.6 $\mathrm{mm} \mathrm{Hg}, \mathrm{p}<0.01)$. Intergroup comparison of changes of SBP from baseline following ingestion of MPP versus comparator revealed significant differences at all measurement time-points, whereas changes of DBP from baseline following supplementation with MPP versus comparator were non-significant at all assessment time-points (Table 1). Following ingestion of MPP, no significant change in heart rate from baseline was noted at 30,60, 90, and 120 minutes post-ingestion whereas following ingestion of comparator, significant reductions of this parameter were noted at 30, 60, 90, and 120 minutes post-ingestion (Table 2). Moreover, significant intergroup differences of change of heart rate from baseline were noted following ingestion of MPP ${ }^{\mathrm{TM}}$ versus comparator at 60,90 , and 120 minutes $(\mathrm{p}<0.008)$.

Table I. Acute safety study: changes in blood pressure from baseline to post-baseline time-points.

\begin{tabular}{|c|c|c|c|}
\hline Parameter (time)/statistic & MPP & Comparator & P-value ${ }^{1}$ \\
\hline SBP (30 minutes) & & & 0.026 \\
\hline Mean (SD), mmHg & $3.4(8.1)$ & $-0.3(6.5)$ & \\
\hline P-value ${ }^{2}$ & 0.012 & 0.753 & \\
\hline SBP (60 minutes) & & & $<0.001$ \\
\hline Mean (SD), mmHg & $4.9(8.2)$ & $-1.2(6.7)$ & \\
\hline $\mathrm{P}$-value ${ }^{2}$ & 0.001 & 0.255 & \\
\hline SBP (90 minutes) & & & 0.001 \\
\hline Mean (SD), mmHg & $5.4(7.7)$ & $-0.6(8.5)$ & \\
\hline P-value ${ }^{2}$ & $<0.001$ & 0.644 & \\
\hline SBP (120 minutes) & & & 0.020 \\
\hline Mean (SD), mmHg & $4.9(8.7)$ & $0.0(9.6)$ & \\
\hline P-value ${ }^{2}$ & 0.001 & 0.987 & \\
\hline DBP (30 minutes) & & & 0.390 \\
\hline Mean (SD), mmHg & $2.3(7.1)$ & $3.6(6.7)$ & \\
\hline P-value ${ }^{2}$ & 0.052 & 0.002 & \\
\hline DBP (60 minutes) & & & 0.446 \\
\hline Mean (SD), mmHg & $4.2(6.1)$ & $3.1(6.1)$ & \\
\hline $\mathrm{P}$-value ${ }^{2}$ & $<0.001$ & 0.003 & \\
\hline DBP (90 minutes) & & & 0.925 \\
\hline Mean (SD), mmHg & $3.7(7.1)$ & $3.9(7.6)$ & \\
\hline P-value ${ }^{2}$ & 0.002 & 0.003 & \\
\hline DBP (120 minutes) & & & 0.405 \\
\hline Mean (SD), $\mathrm{mmHg}$ & $4.3(6.6)$ & $3.0(7.3)$ & \\
\hline $\mathrm{P}$-value ${ }^{2}$ & $<0.001$ & 0.014 & \\
\hline
\end{tabular}

$\mathrm{SBP}$, systolic blood pressure; DBP, diastolic blood pressure.
Table 2. Acute safety study: changes in heart rate from baseline to post-baseline time-points.

\begin{tabular}{|c|c|c|c|}
\hline Parameter (time)/statistic & MPP & Comparator & P-value \\
\hline Heart rate (30 minutes) & & & 0.183 \\
\hline Mean (SD) & $-2.1(7.1)$ & $-4.2(5.8)$ & \\
\hline P-value ${ }^{2}$ & 0.058 & $<0.001$ & \\
\hline Heart rate (60 minutes) & & & 0.003 \\
\hline Mean (SD) & $1.1(8.6)$ & $-3.9(5.8)$ & \\
\hline P-value ${ }^{2}$ & 0.001 & $<0.001$ & \\
\hline Heart rate (90 minutes) & & & 0.008 \\
\hline Mean (SD) & $0.0(8.6)$ & $-4.5(5.5)$ & \\
\hline P-value ${ }^{2}$ & 1.000 & $<0.001$ & \\
\hline Heart rate (120 minutes) & & & 0.005 \\
\hline Mean (SD) & $0.6(8.9)$ & $-5.8(6.7)$ & \\
\hline P-value ${ }^{2}$ & 0.657 & $<0.001$ & \\
\hline
\end{tabular}

Analysis of ECG data extracted for all subjects failed to uncover any significant influence on the primary outcome variable, corrected QT interval (QTc) according to Fridericia's formula. Specifically, the change in QTc value was $2.6 \mathrm{msec}$ in the comparator group (from 411.5 to 414.1 ) and $6.8 \mathrm{msec}$ in the MPP group (from 411.1 to 417.9 ), resulting in no intergroup difference $(\mathrm{p}<0.11)$.

\section{Part 2: Six-week Efficacy Study \\ Subjects}

A total of 43 subjects were recruited and randomized to receive MPP $(n=22)$ or the comparator product $(n=21)$. At baseline, subjects in the two treatment groups were well matched as shown in table 1. From a compliance/attrition perspective, 21 of 22 subjects $(95.5 \%)$ in the MPP group and 18 of 21 subjects $(85.7 \%)$ in the comparator product group completed the study; no difference $(p>0.05)$ was found regarding rates of compliance for each group. No changes across groups and over time were noted in dietary habits (i.e. energy, carbohydrate, protein and fat intake [data not shown]).

\section{Efficacy Biomarkers}

Efficacy analysis revealed consistently higher levels of energy and focus in subjects who took MPP versus those who took the comparator product during all sets of upper body muscular endurance testing (weeks 3 and 6). Significant differences of energy were noted at week 3 (Sets 2 and $3[\mathrm{P}=0.006$ and 0.024 , respectively]) and week 6 (Set $3[\mathrm{P}=0.008]$ ) (figure 2). Meanwhile, significant improvements in concentration were noted in the MPP versus comparator group at week 3 (Set $3[\mathrm{P}=0.041]$ ) and week 6 (Sets 2 and 3 $[\mathrm{P}=0.034$ and 0.040, respectively]) (Fig. 3). Self-perception of focus was higher and fatigue lower 
in the MPP versus comparator group consistently during all sets at weeks 3 and 6, although the differences did not reach statistical significance.

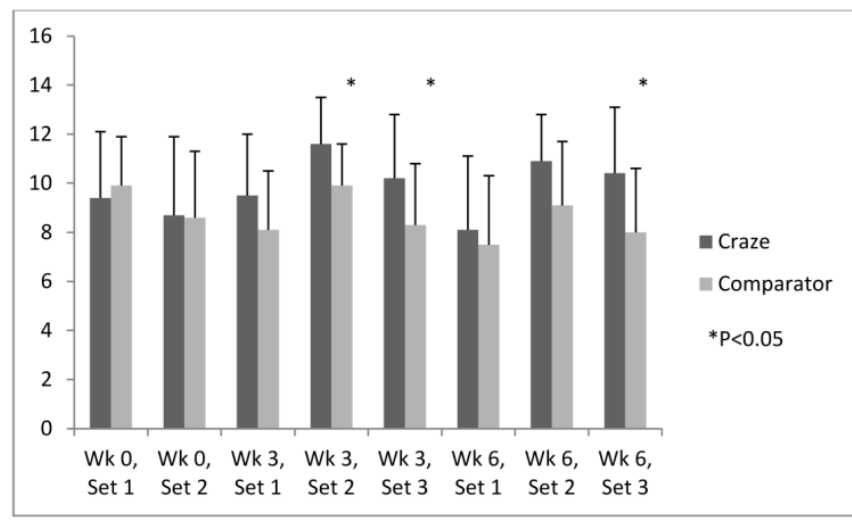

Figure 2. Efficacy endpoints: time-course of energy.

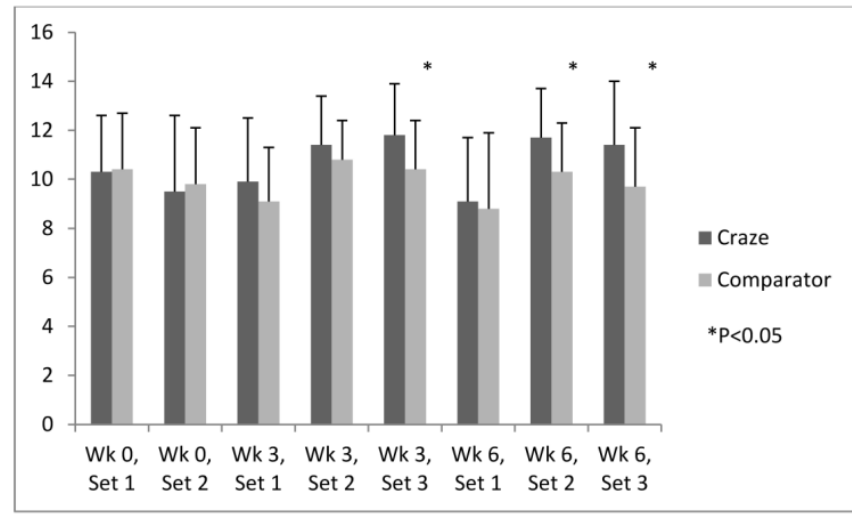

Figure 3. Efficacy endpoints: time-course of concentration.
No statistically significant difference was noted between the MPP and comparator product groups in terms of DEXA-determined tissue mass $(p=0.781)$, fat mass $(p=0.256)$, lean mass $(p=0.351)$, or $L: F$ ratio $(p=0.170)$ at any assessment time-point or changes from baseline after six weeks of training (Table 3). Subjects who took MPP increased their L:F ratio slightly from 4.5 at baseline to 4.7 at week 6 whereas the L:F ratio of subjects in the comparator group remained at 4.0 throughout the study.

No significant intergroup difference was noted in terms of bench press maximum weight (MPP: $9.64 \pm 4.82$ vs. PLA: $8.73 \pm 5 \mathrm{~kg}, \mathrm{p}=0.563$ ) and bench press 1-RM relative to lean mass (MPP: $0.13 \pm 0.01$ vs. PLA: $\quad 0.12 \pm 0.08, \mathrm{p}=0.836)$ at any assessment time-point or changes of these parameters from baseline. No significant intergroup differences were noted in terms of either absolute values of AP (MPP: $378 \pm$ 99 vs. PLA: $403 \pm 119$ watts, $\mathrm{p}=0.262$ ), AV (MPP: $0.88 \pm$ 0.06 vs. PLA: $0.88 \pm 0.10$ meters/second, $p=0.403)$, PP (MPP: $563 \pm 138$ vs. PLA: $593 \pm 189$ watts, $p=0.649$ ), and PV (MPP: $1.31 \pm 0.12$ vs. PLA: $1.30 \pm$ 0.22 meters/second, $p=0.442$ ) at any assessment time-point or their changes from baseline. No significant intergroup differences were noted in RTF at any assessment time-point or changes in this parameter from baseline (MPP: $20.6 \pm 13.4$ vs. PLA: $21.1 \pm$ 12.6 reps, $p=0.151$ ) with the exception of a significantly greater change of RTF versus baseline noted in the MPP group than in the comparator group at week 3 (mean [SD]: MPP group, 3.8 [4.0]; comparator group, -0.9 [8.3]; $\mathrm{P}=0.024)$. Moreover, in the MPP group the change of RTF at week 3 versus baseline was itself statistically significant $(\mathrm{p}<0.01)$.

Table 3. Efficacy endpoints: tissue, fat, lean, and lean:fat (L:F) ratio.

\begin{tabular}{llll}
\hline Parameter & MPP & Comparator & P-value \\
\hline Tissue mass at baseline $(\mathrm{g})$ & $79,160.3(6720.1)$ & $81,144(11,604.2)$ & 0.510 \\
Change of tissue at Week 3 $(\mathrm{g})$ & $464.5(1610.3)$ & 1120.4 & 0.133 \\
Change of tissue at Week 6 (g) & $520.2(1881.9)$ & 696.8 & 0.781 \\
Fat mass at baseline $(\mathrm{g})$ & $16,238.2(5647.4)$ & $17,257.2(5738.9)$ & 0.581 \\
Change of fat at Week 3 (g) & $-386.1(1654.5)$ & 313.9 & 0.223 \\
Change of fat at Week 6 (g) & $-407.2(2275.6)$ & $63,887.7(9125.8)$ & 0.256 \\
Lean mass at baseline (g) & $62,922.2(6417.6)$ & $900.2(1502.8)$ & 0.701 \\
Change of lean at Week 3 (g) & $850.7(1832.9)$ & $382.9(1679.4)$ & 0.928 \\
Change of lean at Week 6 (g) & $927.4(1886.2)$ & $4.0(1.3)$ & 0.351 \\
L:F ratio at baseline & $4.5(2.2)$ & $-0.1(0.4)$ & 0.397 \\
Change of L:F ratio at Week 3 & $0.1(0.5)$ & $-0.1(0.5)$ & 0.211 \\
Change of L:F ratio at Week 6 & $0.2(0.7)$ & & 0.170 \\
\hline
\end{tabular}




\section{Vital Signs and Adverse Events}

Six weeks of daily supplementation with MPP exerted no overall effect on change of vital signs such as blood pressure and heart rate (Table 4). A statistically significant group $x$ time interaction was identified for delta changes in DBP at week 6 (mean [SD]: MPP group, -1.7 [6.9] mmHg; comparator group, +6.9 [12.8] $\mathrm{mmHg} ; \mathrm{P}=0.011$ ). No significant group $\mathrm{x}$ time interaction was found for SBP $(p=0.323)$. In comparison to baseline values, a non-significant decrease $(\mathrm{p}=0.191)$ occurred for SBP within in the MPP group $(-2.2 \pm 7.4 \mathrm{~mm} \mathrm{Hg})$. No other significant differences in vital signs were noted between the groups at weeks 3 and 6.

Table 4. Six-week efficacy study: changes in vital signs from baseline to post-baseline time-points.

\begin{tabular}{|c|c|c|c|}
\hline Parameter & MPP & Comparator & $\begin{array}{l}\text { Group x Time } \\
\text { P-value }\end{array}$ \\
\hline $\begin{array}{l}\text { SBP, Week } 3 \\
(\mathrm{mmHg})\end{array}$ & & & 0.076 \\
\hline Mean (SD) & $3.2(6.6)$ & $-0.8(7.2)$ & \\
\hline $\begin{array}{l}\text { Within Group } \\
\text { P-value }\end{array}$ & 0.033 & 0.654 & \\
\hline $\begin{array}{l}\text { SBP, Week } 6 \\
(\mathrm{mmHg})\end{array}$ & & & 0.323 \\
\hline Mean (SD) & $-2.2(7.4)$ & $0.7(10.7)$ & \\
\hline $\begin{array}{l}\text { Within Group } \\
\text { P-value }\end{array}$ & 0.191 & 0.777 & \\
\hline $\begin{array}{l}\text { DBP, Week } 3 \\
(\mathrm{mmHg})\end{array}$ & & & 0.161 \\
\hline Mean (SD) & $0.6(6.6)$ & $-2.2(5.4)$ & \\
\hline $\begin{array}{l}\text { Within Group } \\
\text { P-value }\end{array}$ & 0.677 & 0.107 & \\
\hline $\begin{array}{l}\text { DBP, Week } 6 \\
(\mathrm{mmHg})\end{array}$ & & & 0.011 \\
\hline Mean (SD) & $-1.7(6.9)$ & $6.9(12.8)$ & \\
\hline $\begin{array}{l}\text { Within Group } \\
\text { P-value }\end{array}$ & 0.267 & 0.035 & \\
\hline HR, Week 6 (bpm) & & & 0.709 \\
\hline Mean (SD) & $2.5(6.9)$ & $1.5(9.3)$ & \\
\hline $\begin{array}{l}\text { Within Group } \\
\text { P-value }\end{array}$ & 0.116 & 0.502 & \\
\hline
\end{tabular}

No statistically significant change of clinical laboratory parameters such as BUN, creatinine, BUN:creatinine ratio, total protein, albumin, globulin, A/G ratio, cholesterol, LDL, HDL, cholesterol:HDL, TG, WBC, RBC, hemoglobin, hematocrit, and CRP was noted at any assessment time-point (all $\mathrm{p}>0.05$ ), apart from a significantly $(\mathrm{P}=0.021)$ lowered blood creatinine level in the comparator group at end of study (week 6). However, despite this reduction the creatinine level in this group remained within the normal clinical reference range. Relative to adverse events, subjects taking MPP experienced the follow- ing: slightly jittery $(n=2)$, more energy $(n=6)$, headache $(n=1)$, upset stomach $(n=2)$ trouble sleeping $(n=1$, for the first week only). Similarly, subjects in the comparator group reported: more energy $(n=2)$, headache $(n=3)$, nervousness $(n=1)$, upset stomach $(n=1)$. None of these differences were statistically significant.

\section{DISCUSSION}

The use of nutritional supplements by competitive athletes and recreationally active fitness enthusiasts is growing increasingly popular $[2,13]$. The category of sports supplements in the "concentrated pre-workout" class includes a bevy of multi-ingredient products typically containing a stimulant blend with caffeine and other alkaloids often including creatine, L-citrulline, and other nitrogenous compounds aimed at augmenting several metabolic pathways for an ergogenic beneficial effect. However, much of the hypothetical usefulness of these supplements is based on data on their individual ingredients rather than finished products. In our study, six weeks of supplementation with MPP in concert with a heavy resistance training program was associated with significantly higher subjective feelings of energy levels and significantly improved concentration over comparator product at weeks 3 and 6 . Moreover, subjective feelings of focus were consistently higher and fatigue consistently lower during all workout sets throughout the study. On the other hand, MPP supplementation resulted in no overall improvement in markers of muscular performance such as upper and lower body power output (AP, AV, PP, PV), muscular endurance (RTF), and strength (1-RM WT, 1-RM/LM) compared with subjects who took a positive control (comparator) product pre-workout.

It is well established that among healthy adults voluntary participation in physical exercise programs is often accompanied by substantial dropout rates of approximately $50 \%$ during the first 6 months $[26,27]$. A study conducted in 66 healthy adult men who were enrolled in a prospective 20-week physical activity program including muscular endurance exercise concluded that self-motivation was highly correlated with exercise adherence irrespective of other possible confounding factors such as gym setting, exercise leader, and interpersonal relationships [26, 27]. It therefore follows that a potential benefit of elevating energy, concentration, and focus and alleviating fatigue during workouts using MPP might be to improve the subjective experience of performing exercise and thereby increase motivation and exercise adherence.

Although MPP exerted no statistically significant beneficial effect over comparator product on total 
tissue, fat, lean mass and L:F ratio as revealed by DEXA, trends were observed for a reduction of fat mass accompanied by an increase of lean mass over the 6-week efficacy study period. Although speculative at this time, it is conceivable that with a longer period for training-supplement interaction, more marked improvements of body composition adaptations to the resistance exercise program may be observed.

Supplementation with MPP was generally safe. No changes in clinical laboratory parameters, serum electrolytes, liver enzymes, blood lipids, or blood cell counts were observed and there were no serious adverse events. In subjects who were supplemented with MPP in part 2 (6-wk) of this study, however, statistically significant reductions of DBP versus baseline were noted at week 6 whereas this parameter was increased in the comparator group. Moreover, SBP in the MPP group were not changed after 6 weeks $(P=0.191)$ as was heart rate, whereas heart rate was significantly reduced at all measurement time-points following ingestion of comparator product. The finding of a lowered SBP and DBP in the 6-week efficacy study is in contrast to that of the acute safety study (Part 1) wherein SBP and DBP were significantly increased up to two hours after taking MPP while SBP remained unchanged and DBP slightly increased in the comparator group. These apparently conflicting results are confounded by the observation that subjects in the MPP and comparator groups alike were supplemented with caffeine-containing pre-workout blends, since caffeine is known to increase blood pressure acutely (i.e. peak values at $\sim 30$ minutes post-oral ingestion and persisting for up to two hours) $[28,29]$. Caffeine is also known to induce tachycardia in some individuals, especially non-habitual consumers [30]. Our results may therefore be attributed to an artifact of measurement procedure and the observed SBP and DBP alterations of approximately 2-5 $\mathrm{mm} \mathrm{Hg}$ do not warrant clinical concern in healthy young adults who were normotensive and exhibit normal sinus rhythm at baseline.

Dietary supplements are highly popular among professional and recreational athletes, as well as fitness enthusiasts as a means of enhancing training adaptations [2, 31]. However, supplement manufacturers often advertise products whose use in sports is neither scientifically founded nor safe, resulting in an irrational use of dietary supplements that are of little benefit, and which may lead to unwanted side effects. The proposed or advertised ergogenic effect of many supplements is based on a presumptive metabolic pathway and may not necessarily translate to quantifiable changes in a variable as broadly defined as exercise performance [32]. The present randomized, double-blind study conducted at a single research site was an initial attempt to investigate the safety and efficacy of the commercially available pre-workout product MPP. More controlled trials should be performed on such widely used products as pre- and post-workout supplements so as better to inform physicians, consumers, trainers, and other researchers of their potential usefulness in this setting.

As with all studies in free-living humans, our study has some limitations. During the chronic (6-week) study, we did not monitor daily supplement ingestion, or have strict control over workouts or dietary intake. We attempted to lessen the impact of these limitations by maintaining constant communication with the participants and requiring them to complete various supplementation, training and diet logs. Our approach to these concerns is consistent with other published studies [33-36]. It is also possible that other changes occurred that our chosen measurement procedures did not detect such as changes in lower body strength, power and endurance. Finally, another fundamental difficulty of conducting studies of multiple physiologically impactful ingredients is that measured outcomes may only be attributed to one ingredient. Certainly conducting multiple arms of an identical study protocol with additional study participants would address this concern, but this is both cost and time-prohibitive and lacks ecological practicality because products such as MPP are extremely popular in the sports nutrition market of the dietary supplement industry.

In summary, pre-workout supplementation with MPP, a proprietary blend containing creatine, TMG, dendrobium extract (Dendrobex ${ }^{\mathrm{TM}}$ ) and caffeine, significantly improved subjective levels of energy and concentration and tended to increase focus and lessen fatigue versus comparator product containing caffeine alone. MPP supplementation bestowed no statistically significant effect on measures of body composition or exercise performance, at least over six weeks of intensive training. By improving the perceived exercise experience, MPP might bolster self-motivation, a known influencing factor in adherence rates to exercise programs. Supplementation with MPP was generally safe and well-tolerated; this pre-workout supplement did not induce any harmful effects on clinically relevant serum biochemistry parameters, systemic hemodynamics, or ECG tracings.

\section{Acknowledgments}

The authors would like to thank the subjects who participated in the study and Alex Kahney of Niche Science and Technology for providing editorial support during the development of this manuscript. The presentation of results of this study does not consti- 
tute endorsement by the any of the investigators, The Center for Applied Health Sciences, or the journal in which it is published. The sponsor of this study, Driven Sports, Inc. (Franklin Square, NY) provided an independent research grant and donated product, but had no role in the collection, analyses, or interpretation of the data.

\section{Authors' contributions}

AWK provided medical oversight, subject screening, subject recruitment and assisted with data collection and manuscript preparation. HLL designed the study, secured funding for project, assisted with data analysis and manuscript preparation. JH and SMH carried out subject recruitment, data collection, exercise training, coordination of the study and compliance. MDG was responsible for data analysis and interpretation. AAF assisted with data analysis and manuscript preparation. All authors read and approved the final manuscript.

\section{Competing Interests}

The authors declare that they have no competing or financial interests concerning the outcome of this investigation. The sponsor of this study, Driven Sports, Inc. (Franklin Square, NY) provided an independent research grant and donated product, but had no role in the collection, analyses, or interpretation of the data.

\section{References}

1. Kerksick C, Harvey T, Stout J, Campbell B, Wilborn C, Kreider R, et al. International Society of Sports Nutrition position stand: nutrient timing. J Int Soc Sports Nutr. 2008; 5: 17. doi:10.1186/1550-2783-5-17.

2. Kreider RB, Wilborn CD, Taylor L, Campbell B, Almada AL, Collins R, et al. ISSN exercise \& sport nutrition review: research \& recommendations. J Int Soc Sports Nutr. 2010; 7: 7. doi:10.1186/1550-2783-7-7.

3. Fukuda DH, Smith AE, Kendall KL, Stout JR. The possible combinatory effects of acute consumption of caffeine, creatine, and amino acids on the improvement of anaerobic running performance in humans. Nutrition research. 2010; 30: 607-14. doi:10.1016/j.nutres.2010.09.004.

4. Kraemer WJ, Hatfield DL, Spiering BA, Vingren JL, Fragala MS, Ho JY, et al. Effects of a multi-nutrient supplement on exercise performance and hormonal responses to resistance exercise. Eur J Appl Physiol. 2007; 101: 637-46. doi:10.1007/s00421-007-0535-3.

5. Smith AE, Fukuda DH, Kendall KL, Stout JR. The effects of a pre-workout supplement containing caffeine, creatine, and amino acids during three weeks of high-intensity exercise on aerobic and anaerobic performance. J Int Soc Sports Nutr. 2010; 7: 10. doi:10.1186/1550-2783-7-10.

6. Spillane M, Schwarz N, Leddy S, Correa T, Minter M, Longoria V, et al. Effects of 28 days of resistance exercise while consuming commercially available preand post-workout supplements, NO-Shotgun(R) and NO-Synthesize(R) on body composition, muscle strength and mass, markers of protein synthesis, and clinical safety markers in males. Nutr Metab (Lond). 2011; 8: 78. doi:10.1186/1743-7075-8-78.

7. Buford TW, Kreider RB, Stout JR, Greenwood M, Campbell B, Spano M, et al. International Society of Sports Nutrition position stand: creatine supplementation and exercise. J Int Soc Sports Nutr. 2007; 4: 6. doi:10.1186/1550-2783-4-6.

8. Greenhaff PL. Muscle creatine loading in humans: Procedures and functional and metabolic effects. Cincinnati, $\mathrm{OH}$ : 6th International Conference on Guanidino Compounds in Biology and Medicine. 2001.

9. Kreider RB, Ferreira M, Wilson M, Grindstaff P, Plisk S, Reinardy J, et al. Effects of creatine supplementation on body composition, strength, and sprint performance. Medicine and science in sports and exercise. 1998; 30: 73-82.

10. Cribb PJ, Hayes A. Effects of supplement timing and resistance exercise on skeletal muscle hypertrophy. Med Sci Sports Exerc. 2006; 38: 1918-25. doi:10.1249/01.mss.0000233790.08788.
11. Kreider R.B., et al. Long-term creatine supplementation does not significantly affect clinical markers of health in athletes. Mol Cell Biochem, 2003. 244(1-2): 95-104.

12. Trepanowski JF, Farney TM, McCarthy CG, Schilling BK, Craig SA, Bloomer RJ. The effects of chronic betaine supplementation on exercise performance, skeletal muscle oxygen saturation and associated biochemical parameters in resistance trained men. J Strength Cond Res. 2011; 25: 3461-71. doi:10.1519/JSC.0b013e318217d48d.

13. Maughan RJ. Nutritional ergogenic aids and exercise performance. Nutr Res Rev. 1999; 12: 255-80. doi:10.1079/095442299108728956.

14. Astorino TA, Roberson DW. Efficacy of acute caffeine ingestion for short-term high-intensity exercise performance: a systematic review. J Strength Cond Res. 2010; 24: 257-65. doi:10.1519/JSC.0b013e3181c1f88a.

15. Williams MH. Facts and fallacies of purported ergogenic amino acid supplements. Clin Sports Med. 1999; 18: 633-49.

16. Davis JK, Green JM. Caffeine and anaerobic performance: ergogenic value and mechanisms of action. Sports Med. 2009; 39: 813-32. doi:10.2165/11317770-000000000-00000.

17. Doherty $\mathrm{M}$ and Smith P.M. Effects of caffeine ingestion on rating of perceived exertion during and after exercise: a meta-analysis. Scand J Med Sci Sports, 2005. 15(2): 69-78.

18. Takamiya T, Wongsawad P, Tajima N, Shioda N, Lu JF, Wen CL, et al. Identification of dendrobium species used for herbal medicines based on ribosomal DNA internal transcribed spacer sequence. Biol Pharm Bull. 2011; 34: 779-82.

19. Kerksick CM, Wilborn CD, Campbell BI, Roberts MD, Rasmussen CJ, Greenwood M, et al. Early-phase adaptations to a split-body, linear periodization resistance training program in college-aged and middle-aged men. J Strength Cond Res. 2009; 23: 962-71. doi:10.1519/JSC.0b013e3181a00baf.

20. Ziegenfuss TN, Hofheins JE, Mendel RW, Landis J, Anderson RA. Effects of a water-soluble cinnamon extract on body composition and features of the metabolic syndrome in pre-diabetic men and women. J Int Soc Sports Nutr. 2006; 3: 45-53. doi:10.1186/1550-2783-3-2-45.

21. Baechle TR, Earle RW. Essentials of Strength Training and Conditioning. Champaign, IL: Human Kinetics; 2000.

22. Alemany JA, Pandorf CE, Montain SJ, Castellani JW, Tuckow AP, Nindl BC. Reliability assessment of ballistic jump squats and bench throws. J Strength Cond Res. 2005; 19: 33-8. doi:10.1519/14783.1.

23. Hoffman JR, Ratamess NA, Kang J, Rashti SL, Faigenbaum AD. Effect of betaine supplementation on power performance and fatigue. J Int Soc Sports Nutr. 2009; 6: 7. doi:10.1186/1550-2783-6-7.

24. Stock MS, Beck TW, DeFreitas JM, Dillon MA. Test-retest reliability of barbell velocity during the free-weight bench-press exercise. J Strength Cond Res. 2011; 25: 171-7. doi:10.1519/JSC.0b013e318201bdf9.

25. Lee KA, Hicks G, Nino-Murcia G. Validity and reliability of a scale to assess fatigue. Psychiatry Res. 1991; 36: 291-8.

26. Ice R. Long-term compliance. Phys Ther. 1985; 65: 1832-9.

27. Robison JI, Rogers MA. Adherence to exercise programmes. Recommendations. Sports Med. 1994; 17: 39-52.

28. Mort JR, Kruse HR. Timing of blood pressure measurement related to caffeine consumption. Ann Pharmacother. 2008; 42: 105-10. doi:10.1345/aph.1K337.

29. Nurminen ML, Niittynen L, Korpela R, Vapaatalo H. Coffee, caffeine and blood pressure: a critical review. Eur J Clin Nutr. 1999; 53: 831-9.

30. Tarnopolsky MA. Caffeine and creatine use in sport. Ann Nutr Metab. 2010; 57 Suppl 2: 1-8. doi:10.1159/000322696.

31. Koncic MZ, Tomczyk M. New insights into dietary supplements used in sport: active substances, pharmacological and side effects. Curr Drug Targets. 2013; 14: 1079-92.

32. Paddon-Jones D, Borsheim E, Wolfe RR. Potential ergogenic effects of arginine and creatine supplementation. J Nutr. 2004; 134: 2888S-94S; discussion 95S

33. Campbell B, Roberts M, Kerksick C, Wilborn C, Marcello B, Taylor L, et al. Pharmacokinetics, safety, and effects on exercise performance of L-arginine alpha-ketoglutarate in trained adult men. Nutrition. 2006; 22: 872-81. doi:10.1016/j.nut.2006.06.003.

34. Kerksick CM, Rasmussen CJ, Lancaster SL, Magu B, Smith P, Melton C, et al. The effects of protein and amino acid supplementation on performance and training adaptations during ten weeks of resistance training. J Strength Cond Res. 2006; 20: 643-53. doi:10.1519/R-17695.1.

35. Roberts MD, Iosia M, Kerksick CM, Taylor LW, Campbell B, Wilborn CD, et al. Effects of arachidonic acid supplementation on training adaptations in resistance-trained males. J Int Soc Sports Nutr. 2007; 4: 21. doi:10.1186/1550-2783-4-21.

36. Wilborn CD, Taylor LW, Campbell BI, Kerksick C, Rasmussen CI, Greenwood $\mathrm{M}$, et al. Effects of methoxyisoflavone, ecdysterone, and sulfo-polysaccharide supplementation on training adaptations in resistance-trained males. J Int Soc Sports Nutr. 2006; 3: 19-27. doi:10.1186/1550-2783-3-2-19. 